\title{
PRINTER'S ERROR
}

In Vol. VII, No. 2, the pagination is not continuous, pages $147^{-1} 54$ being missing from the numbering.

Antiq. Fourn. 